\title{
Polymorphism and C-H...O Interaction of Wortmannin, a Phosphatidylinositol 3-Kinase Inhibitor
}

\author{
Mitsunobu DoI*, Toshimasa IshIDA*, Akira Terashima**, Taizo TANiguchI**, \\ Masahiro SASAKI** and Chikako TANAKA** \\ * Osaka University of Pharmaceutical Sciences, Nasahara, Takatsuki, Osaka 569-1094, Japan \\ **Hyogo Institute for Aging Brain and Cognitive Disorders, Saisho-ko, Himeji 670-0981, Japan
}

Wortmannin is isolated from Penicillium wortmanni and is a potent inhibitor of phosphatidylinositol 3kinase (PI 3-kinase). The electrophilic C21-site makes a covalent linkage with the enzyme, and the inhibition is irreversible (Fig. 1). PI 3-kinase mediates several intracellular signaling pathways, and wortmannin has been examined as a probe to understand the structural requirements necessary for PI 3-kinase inhibition. ${ }^{1}$ Two related structures have been reported for wortmannin: one is an orthorhombic crystal with space group $P 2{ }_{1} 2_{1} 2_{1} ;{ }^{2}$ the other is a C21-methylated analogue, of which the crystal is monoclinic and $P 2_{1}{ }^{1}$ We report a polymorphic structure of wortmannin with a monoclinic system and space group $C 2$.

Needle-shaped crystals with the size $0.70 \times 0.08 \times 0.08$ $\mathrm{mm}^{3}$ were obtained from an aqueous methanol solution.

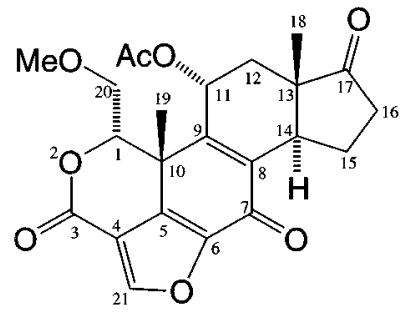
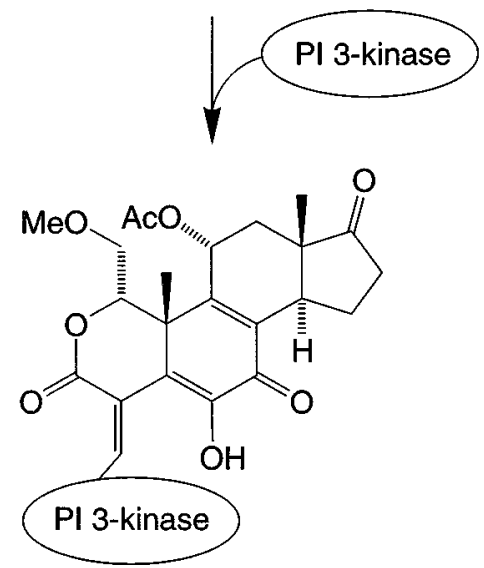

Fig. 1 Chemical structure of wortmannin and a proposed inhibition mechanism.
The intensity data were collected to approximately $2 \theta=130^{\circ}$ in the $\omega-2 \theta$ scan mode using graphite-monochromated $\mathrm{Cu} \mathrm{K} \alpha$ radiation $(\lambda=1.5418 \AA)$. This compound is sensitive against light, though no change in the crystalline appearance was observed during X-ray exposure. The data were corrected for Lorentz and polarization factors, but no absorption correction was applied. Table 1 summarizes the crystal and experimental data. The structure was solved by a direct method using SHELXS-97, and a full-matrix leastsquares method was applied for a refinement using SHELXL-97. Non-hydrogen atoms were refined anisotropically. Hydrogen atoms calculated at idealized positions were included in the calculation of the structure factors. The atomic parameters are given in Table 2. The bond distances and angles are given in Table 3 .

A short contact is found at $\mathrm{C} 21 \cdots \mathrm{O} 20(3 / 2-x, 1 / 2+y, 1-z)$; $\mathrm{C} 21-\mathrm{H} 21=0.930, \mathrm{H} 21 \cdots \mathrm{O} 20=2.205, \mathrm{C} 21 \cdots \mathrm{O} 20=3.096(4)$

Table 1 Crystal and experimental data

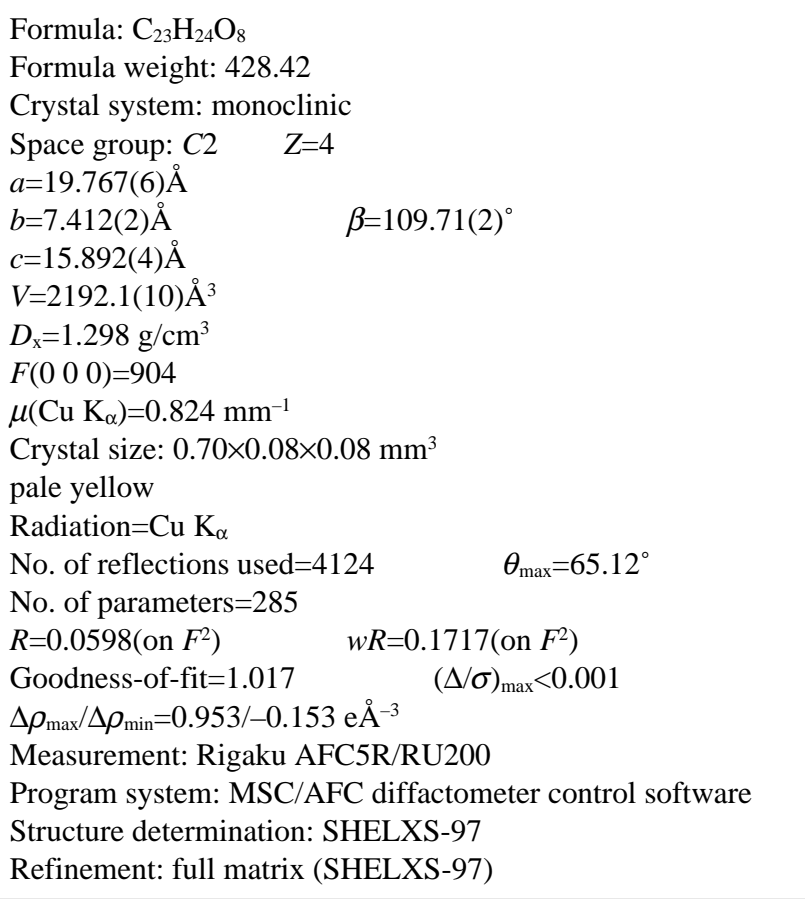


Table 2 Final atomic coordinates and equivalent isotropic temperature factors for non-hydrogen atoms

\begin{tabular}{lllll}
\hline Atom & $x$ & $y$ & $z$ & $U_{\mathrm{eq}} / \mathrm{A}^{2}$ \\
\hline $\mathrm{C} 1$ & $0.6626(1)$ & 0.7361 & $0.3715(2)$ & $0.0461(6)$ \\
$\mathrm{O} 2$ & $0.70898(9)$ & $0.7183(3)$ & $0.4652(1)$ & $0.0544(5)$ \\
$\mathrm{C} 3$ & $0.7067(1)$ & $0.8326(4)$ & $0.5302(2)$ & $0.0519(6)$ \\
$\mathrm{O} 3$ & $0.7540(1)$ & $0.8291(4)$ & $0.6018(1)$ & $0.0708(6)$ \\
$\mathrm{C} 4$ & $0.6432(1)$ & $0.9482(4)$ & $0.5049(2)$ & $0.0486(6)$ \\
$\mathrm{C} 5$ & $0.5858(1)$ & $0.9137(4)$ & $0.4236(2)$ & $0.0443(6)$ \\
$\mathrm{C} 6$ & $0.5418(1)$ & $1.0585(4)$ & $0.4084(2)$ & $0.0470(6)$ \\
$\mathrm{C} 7$ & $0.4888(1)$ & $1.0936(4)$ & $0.3230(2)$ & $0.0474(6)$ \\
$\mathrm{O} 7$ & $0.4528(1)$ & $1.2312(3)$ & $0.3051(2)$ & $0.0692(6)$ \\
$\mathrm{C} 8$ & $0.4848(1)$ & $0.9494(4)$ & $0.2550(2)$ & $0.0422(6)$ \\
$\mathrm{C} 9$ & $0.5284(1)$ & $0.8015(4)$ & $0.2716(2)$ & $0.0405(5)$ \\
$\mathrm{C} 10$ & $0.5828(1)$ & $0.7604(4)$ & $0.3649(2)$ & $0.0421(6)$ \\
$\mathrm{C} 11$ & $0.5182(1)$ & $0.6525(4)$ & $0.2026(2)$ & $0.0470(6)$ \\
$\mathrm{O} 11$ & $0.5885(1)$ & $0.6048(3)$ & $0.2004(1)$ & $0.0533(5)$ \\
$\mathrm{O} 11 \mathrm{~A}$ & $0.5516(2)$ & $0.3253(5)$ & $0.1571(3)$ & $0.120(1)$ \\
$\mathrm{C} 11 \mathrm{~A}$ & $0.5977(2)$ & $0.4359(5)$ & $0.1753(2)$ & $0.0701(9)$ \\
$\mathrm{C} 12 \mathrm{~A}$ & $0.6730(2)$ & $0.4150(7)$ & $0.1735(3)$ & $0.086(1)$ \\
$\mathrm{C} 12$ & $0.4699(2)$ & $0.6988(5)$ & $0.1080(2)$ & $0.0607(8)$ \\
$\mathrm{C} 13$ & $0.4064(1)$ & $0.8046(5)$ & $0.1119(2)$ & $0.0544(7)$ \\
$\mathrm{C} 14$ & $0.4329(2)$ & $0.9818(4)$ & $0.1612(2)$ & $0.0522(6)$ \\
$\mathrm{C} 15$ & $0.3637(2)$ & $1.0954(6)$ & $0.1417(3)$ & $0.080(1)$ \\
$\mathrm{C} 16$ & $0.3241(2)$ & $1.0488(8)$ & $0.0430(3)$ & $0.097(1)$ \\
$\mathrm{C} 17$ & $0.3544(2)$ & $0.8723(7)$ & $0.0242(2)$ & $0.075(1)$ \\
$\mathrm{O} 17$ & $0.3374(2)$ & $0.7961(7)$ & $-0.0469(2)$ & $0.118(1)$ \\
$\mathrm{C} 18$ & $0.3604(2)$ & $0.6944(6)$ & $0.1542(3)$ & $0.0735(9)$ \\
$\mathrm{C} 19$ & $0.5581(2)$ & $0.5888(5)$ & $0.4025(2)$ & $0.0607(8)$ \\
$\mathrm{C} 20$ & $0.6894(1)$ & $0.8855(4)$ & $0.3269(2)$ & $0.0494(6)$ \\
$\mathrm{O} 20$ & $0.7564(1)$ & $0.8294(4)$ & $0.3215(2)$ & $0.0685(6)$ \\
$\mathrm{C} 20 \mathrm{M}$ & $0.7837(2)$ & $0.9513(6)$ & $0.2735(2)$ & $0.0733(9)$ \\
$\mathrm{C} 21$ & $0.6288(2)$ & $1.1096(5)$ & $0.5336(2)$ & $0.0547(7)$ \\
$\mathrm{O} 21$ & $0.5671(1)$ & $1.1814(3)$ & $0.4764(1)$ & $0.0557(5)$ \\
\hline & & & &
\end{tabular}

$U_{\mathrm{eq}}=(1 / 3) \Sigma_{i} \Sigma_{j} U_{i j}\left(a_{i} * a_{j}^{*}\right)\left(\boldsymbol{a}_{i} \cdot \boldsymbol{a}_{j}\right)$.

$\AA$ A, $\angle \mathrm{C} 21-\mathrm{H} 21 \cdots \mathrm{O} 20=160.3^{\circ}$. The distance between $\mathrm{H} 21$ and $\mathrm{O} 20$ atoms is about $0.5 \AA$ below the sum of the van der Waals radii of hydrogen and oxygen. Since there is no abnormal stress in the bonds and angles related to C21 (Table 3), this interaction could be defined as the $\mathrm{C}-\mathrm{H} \cdots \mathrm{O}$ hydrogen bond which is often reported in protein and nucleic acid structures. ${ }^{3}$

An outstanding characteristic of wortmannin is the electrophilic reaction center at the C21-position. The electron state at this position seems to associate with the reactivity of wortmannin and the $\mathrm{C}-\mathrm{H} \cdots \mathrm{O}$ hydrogen bond, because no activity for PI 3-kinase and no abnormal short contact have been reported for the $\mathrm{C} 21$ methylated analogue. ${ }^{1}$
Table 3 Selected bond distances $(\AA)$ and angles $\left({ }^{\circ}\right)$

\begin{tabular}{llll}
\hline $\mathrm{C} 1-\mathrm{C} 10$ & $1.556(4)$ & $\mathrm{C} 6-\mathrm{C} 7$ & $1.431(4)$ \\
$\mathrm{O} 2-\mathrm{C} 3$ & $1.348(3)$ & $\mathrm{C} 6-\mathrm{O} 21$ & $1.372(3)$ \\
$\mathrm{C} 3-\mathrm{O} 3$ & $1.205(4)$ & $\mathrm{C} 7-\mathrm{O} 7$ & $1.221(4)$ \\
$\mathrm{C} 3-\mathrm{C} 4$ & $1.460(4)$ & $\mathrm{C} 7-\mathrm{C} 8$ & $1.504(4)$ \\
$\mathrm{C} 4-\mathrm{C} 5$ & $1.427(4)$ & $\mathrm{C}-\mathrm{C} 10$ & $1.540(4)$ \\
$\mathrm{C} 4-\mathrm{C} 21$ & $1.344(4)$ & $\mathrm{C} 10-\mathrm{C} 19$ & $1.552(4)$ \\
$\mathrm{C} 5-\mathrm{C} 6$ & $1.350(4)$ & $\mathrm{C} 21-\mathrm{O} 21$ & $1.359(4)$ \\
$\mathrm{C} 5-\mathrm{C} 10$ & $1.458(4)$ & & \\
& & & \\
$\mathrm{O} 2-\mathrm{C} 3-\mathrm{O} 3$ & $119.6(2)$ & $\mathrm{C} 6-\mathrm{C} 7-\mathrm{O} 7$ & $123.8(2)$ \\
$\mathrm{O} 2-\mathrm{C} 3-\mathrm{C} 4$ & $114.0(2)$ & $\mathrm{C} 6-\mathrm{C} 7-\mathrm{C} 8$ & $113.4(2)$ \\
$\mathrm{O} 3-\mathrm{C} 3-\mathrm{C} 4$ & $126.4(2)$ & $\mathrm{O} 7-\mathrm{C} 7-\mathrm{C} 8$ & $122.7(2)$ \\
$\mathrm{C} 3-\mathrm{C} 4-\mathrm{C} 5$ & $119.4(2)$ & $\mathrm{C} 1-\mathrm{C} 10-\mathrm{C} 5$ & $103.0(2)$ \\
$\mathrm{C} 3-\mathrm{C} 4-\mathrm{C} 21$ & $133.7(2)$ & $\mathrm{C} 1-\mathrm{C} 10-\mathrm{C} 9$ & $116.8(1)$ \\
$\mathrm{C} 5-\mathrm{C} 4-\mathrm{C} 21$ & $105.8(2)$ & $\mathrm{C} 1-\mathrm{C} 10-\mathrm{C} 19$ & $108.8(2)$ \\
$\mathrm{C} 4-\mathrm{C} 5-\mathrm{C} 6$ & $106.7(2)$ & $\mathrm{C} 5-\mathrm{C} 10-\mathrm{C} 9$ & $109.1(1)$ \\
$\mathrm{C} 4-\mathrm{C} 5-\mathrm{C} 10$ & $124.6(2)$ & $\mathrm{C} 5-\mathrm{C} 10-\mathrm{C} 19$ & $110.0(2)$ \\
$\mathrm{C} 6-\mathrm{C} 5-\mathrm{C} 10$ & $128.3(2)$ & $\mathrm{C} 9-\mathrm{C} 10-\mathrm{C} 19$ & $109.0(2)$ \\
$\mathrm{C} 5-\mathrm{C} 6-\mathrm{C} 7$ & $122.3(2)$ & $\mathrm{C} 4-\mathrm{C} 21-\mathrm{O} 21$ & $11.5(2)$ \\
$\mathrm{C} 5-\mathrm{C} 6-\mathrm{O} 21$ & $110.2(2)$ & $\mathrm{C} 6-\mathrm{O} 21-\mathrm{C} 21$ & $105.8(2)$ \\
$\mathrm{C} 7-\mathrm{C} 6-\mathrm{O} 21$ & $125.5(2)$ & & \\
\hline
\end{tabular}

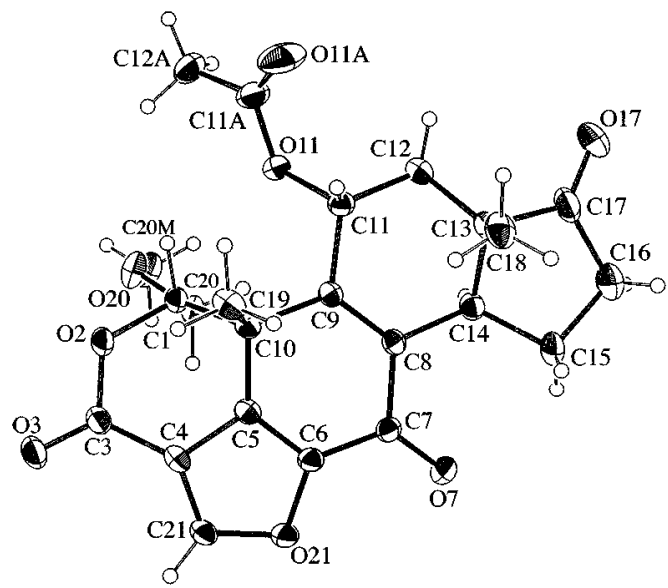

Fig. 2 An ORTEP drawing of the title compound with the atom labeling. The displacement ellipsoids are drawn at 50\% probability level.

\section{References}

1. H. Norman, C. Shih, J. E. Toth, J. E. Ray, J. A. Dodge, D. W. Johnson, P. G. Rutherford, R. M. Schultz, J. F. Worzalla and C. J. Vlahos, J. Med. Chem., 39, 1106 (1996).

2. J. Petcher, H.-P. Weber and Z. Kis, J. Chem. Soc., Chem. Comm., 1972, 1061.

3. M. C. Wahl and M. Sundaralingram, J. Biochem. Sci., 22, 97 (1997).

(Received June 29, 1998) (Accepted August 27, 1998) 